\title{
AUFSÄTZE
}

Werner Distler/Sarah Riese

\section{Die andere Seite der Medaille: Intervention und Widerstand in Bosnien-Herzegowina und im Kosovo}

Interventionen wollen Friedensprozesse, Staatsaufbau und Demokratisierung voranbringen. Die intervenierten Gesellschaften sehen sich jedoch dabei mit den Gestaltungsansprüchen der Intervenierenden konfrontiert. Diese sind der intervenierten Bevölkerung gegenüber weder demokratisch legitimiert, noch unterliegen sie einer Rechenschaftspflicht. Auch institutionalisierte Kanäle zur politischen Konfliktaustragung fehlen. Stattdessen wird Widerstand als Spoilertum delegitimiert. Für uns wird politischer Widerstand deshalb eine erwartbare Folge andauernder Interventionen - die andere Seite der Medaille. Anhand der Partei Savez Nezavisnih Socijaldemokrata aus Bosnien und der Bewegung VETËVENDOSJE! im Kosovo zeigen wir, wie stark sich beide Organisationen als Träger des Widerstands gegen die Interventionen verstehen und sich nur unter deren spezifischen Bedingungen entwickeln konnten. Anstatt Widerstand generell zu delegitimieren und den engen Zusammenhang zwischen Intervention und den aus ihr entstehenden Konflikten zu ignorieren, müssen wir Interventionen deshalb verstärkt als politische Prozesse verstehen, die politische Formen der Konfliktregelung zwischen Intervenierenden und Intervenierten nötig machen.

Schlagworte: Intervention, Statebuilding, Widerstand, Konfliktregelung, Bosnien-Herzegowina, Kosovo

\section{Einleitung}

Internationale Interventionen wollen mittels internationaler Aufsicht Friedensprozesse, Staatsaufbau und Demokratisierung voranbringen. Die intervenierten Gesellschaften sehen sich in diesem Prozess vor allem mit internationalen Präferenzen konfrontiert. Dabei sind die internationalen Akteure der Bevölkerung gegenüber weder demokratisch legitimiert, noch unterliegen sie einer substanziellen Rechenschaftspflicht, kurz: Es fehlt ein Herrschaftsvertrag zwischen Intervenierenden und 
Intervenierten. Dies wird besonders offensichtlich, wenn die Akteure in politischen Prozessen konflikthaft miteinander in Beziehung treten: In der Regel sehen die Planungen der Intervenierenden keine institutionalisierten Kanäle vor, die legitime Kritik ermöglichen (Narten 2007; Zaum 2007). Stattdessen wird Widerstand gegen die Intervention als Spoilertum, also als Gefahr für Frieden, delegitimiert. ${ }^{1}$

Für uns wird unter diesen Bedingungen politischer Widerstand eine erwartbare Folge andauernder Interventionen - Widerstand ist die andere Seite der Medaille. Anhand der Untersuchung zweier Organisationen, der Partei Savez Nezavisnih Socijaldemokrata (SNSD) aus Bosnien-Herzegowina ${ }^{2}$ und der Bewegung VETËVENDOSJE! im Kosovo, zeigen wir, wie beide sich in ihrer Argumentation und ihrem Handeln gegenüber den Intervenierenden positionieren und Intervention in Frage stellen. Die Entwicklung beider Bewegungen konnte nur unter den spezifischen Bedingungen der Intervention erfolgen. In der Analyse zeigen wir zudem, dass die Folgen von Widerstand vielfältig sein können und nicht vorschnell als negativ dargestellt werden sollten: Widerstand ist mehr als nur die Ablehnung der Intervention; er kann auch integrative Folgen für die Gesellschaften haben. Auf der Suche nach den Bedingungen erfolgreicher Interventionen sollten internationale Akteure und wissenschaftliche Literatur gleichermaßen den engen Zusammenhang zwischen Intervention und Widerstand verstehen und reflektieren, anstatt politischen Widerstand zu delegitimieren. Nur dann können Interventionen stärker als bisher als Aushandlungsprozesse verstanden werden, in denen geregelte Formen der politischen Konfliktaustragung notwendig sind.

\section{Die Intervention als politischer Prozess}

Interventionen können als Interaktionsbeziehungen zwischen internationalen und lokalen Akteuren verstanden werden, ob in strategischen Aushandlungsprozessen (Barnett/Zürcher 2009) oder auch im Alltag der Intervention (Distler 2010; Richmond 2009; Pouligny 2006). Für einen begrenzten Zeitraum entstehen gemeinsame Interventionsgesellschaften (Daxner et al. 2010). Die politischen Prozesse, in denen Akteure miteinander in Aushandlungsprozessen stehen, können dabei konfliktbehaftet sein (Bonacker 2010), umso mehr, wenn man sich die Ziele der Intervention vor Augen hält: Die institutionellen und ideellen Grundlagen ganzer Gesellschaften sollen transformiert werden. Dabei ist mitnichten selbstverständlich, dass Intervenierende und Intervenierte dieselben Ziele verfolgen (Zürcher et al. 2013). In In-

1 Wir danken Michael Daxner, Christoph Zürcher, Franziska Smolnik, Sina Schüssler, Laura Vollmann und zwei anonymen Gutachtern für Kommentare und Unterstützung.

2 Im Folgenden wird Bosnien oder $\mathrm{BiH}$ verwendet. 
terventionen entstehen Konflikte sowohl um grundsätzliche Fragen (sind neue Verfassungen, Wahlsysteme, Wirtschaftssysteme notwendig?) wie auch um die konkrete Ausgestaltung dieser institutionellen und ideellen Grundlagen. In diesen Prozessen müssen interne politische Akteure ständig reagieren, ihre und die Interessen ihrer Unterstützer und Wähler einbringen und wahren. Wenn Interventionen immer auch Aushandlungsprozesse sind, stellt sich jedoch die Frage, auf welchen Wegen Konflikte zwischen Intervenierenden und Intervenierten ausgehandelt werden können.

\subsection{Ist geregelte Konfliktaustragung zwischen Intervenierenden und Intervenierten möglich?}

In Interventionsgesellschaften gibt es zunächst keine formalen und informellen Institutionen $^{3}$ (Zürcher/Koehler 2003: 15) zur Bearbeitung von politischen Konflikten zwischen Intervenierenden und Intervenierten. Diese Leerstelle liegt darin begründet, wie Interventionen legitimiert und organisiert werden:

Erstens sind die Intervenierenden meist nur nach außen, nicht aber den intervenierten Gesellschaften gegenüber rechenschaftspflichtig (Chesterman 2004). Zwar müssen Interventionen doppelt legitimiert werden, einerseits auf internationaler Ebene und andererseits gegenüber den intervenierten Gesellschaften (Knoll 2007: 4) - eine formale Legitimierung erfolgt jedoch nur international, meist durch Mandate des UN-Sicherheitsrats. In den intervenierten Gesellschaften versuchen die internationalen Akteure dann Demokratie schlussendlich durchzusetzen, ohne dass die handelnden intervenierenden Organisationen in der intervenierten Gesellschaft demokratisch legitimiert würden (Chandler 1999; Chesterman 2004). Dies wird mit der vermeintlichen Unfähigkeit der intervenierten Gesellschaft, sich selbst zu regieren, begründet (Hughes/Pupavac 2005; Pugh 2000: 3). Mittels unterschiedlicher Konsultationsmechanismen werden die Intervenierten zwar eingebunden, tatsächliche Rechenschaftspflicht entsteht so aber nicht. Mit anderen Worten: In der Intervention gibt es keinen Herrschafts- oder Gesellschaftsvertrag zwischen Intervenierten und Intervenierenden (Chesterman 2011). Der Herrschaftsanspruch der Intervenierenden kann indes als absolut verstanden werden. Selbst nach dem Entstehen neuer politischer Institutionen und Wahlen bestehen sie auf Veto- oder Exekutivrechte; lokale Politik steht unter Vorbehalt.

Zweitens können sich Interventionen weder auf Traditionen berufen noch können sie Legitimität durch demokratische Verfahren herstellen: Sie müssen deshalb auf

3 Institutionen werden hier verstanden als »eine Erwartung über die Einhaltung bestimmter Regeln, die verbindliche Geltung beanspruchen « (Esser 1999: 2; Hervorh. im Orig.). 
diskursive Strategien zurückgreifen und Begründungen liefern, warum die Intervention gerechtfertigt ist (Steffek 2003). Dabei berufen sie sich auf universelle Werte, die sie frei von Eigeninteressen vertreten (Zaum 2007: 58). Der universelle Anspruch der Interventionen wurde treffend als das Paradigma des »liberal peace« identifiziert. ${ }^{4}$ Die Intervenierenden reklamieren für sich das Recht, im Dienste der Gesellschaft die Ziele und Inhalte von Intervention festzulegen (Pugh 2000: 3), quasi in einem selbstlosen Akt (Guilhot 2005). Dies hat gravierende Folgen: Die Ziele der Intervention sind nicht verhandelbar. Zu verhandeln sind, wenn überhaupt, Teilaspekte des Wie und Wann. Es erscheint dann schlicht unnötig, legitime Kanäle für die Aushandlung von Konflikten zu schaffen. Zwar bleibt oberstes Ziel der Intervention stets auch ownership, also die Aneignung der neu geschaffenen Ordnung durch die Bevölkerung und die politischen Entscheidungsträger (Chesterman 2007) - dies soll aber konfliktarm und pragmatisch ablaufen, mehr ein technischer als ein politischer Prozess sein (Wesley 2008).

Drittens gelten Interventionen als außergewöhnliche Antworten auf außergewöhnliche Situationen und sind deshalb konzeptionell immer auf Kurzfristigkeit angelegt (Knoll 2007). Im Normalfall würde eine solche Einmischung in die inneren Angelegenheiten eines Staates gegen internationale Normen der Souveränität verstoßen (Krasner 2007). Das ist nach gängiger Sichtweise nur dann erlaubt, wenn gravierende Gewaltkonflikte und Menschenrechtsverletzungen es notwendig erscheinen lassen. Demnach sind Interventionen aber auch nur so lange legitim, wie diese Gefahr anhält oder die Erwartung diskursiv vermittelbar ist, dass die Beendigung der Intervention erneute Instabilität zur Folge hat. Weil Interventionen darauf angelegt sind, temporär begrenzt zu sein, bewahren sie sich in ihrer Ausgestaltung einen ad-hoc-Charakter. Dieser verbietet schon qua Design, auf Dauer angelegte Institutionen zur Konfliktaustragung zu etablieren - obwohl Interventionen empirisch häufig zu dauerhaften Prozessen werden.

Obwohl Konflikte und Aushandlungsprozesse zum Alltag zwischen Intervenierenden und Intervenierten gehören, lässt sich also zusammenfassend festhalten, dass die Konzeption wie auch die Umsetzung von Intervention kaum Kanäle vorsieht, durch welche diese Konflikte verregelt ausgetragen werden können. Unter diesen Bedingungen entsteht Widerstand, wie im Folgenden gezeigt wird.

4 Liberales Peacebuilding beinhaltet den Aufbau liberaler Demokratie, Marktwirtschaft und Rechtsstaatlichkeit, weil davon ausgegangen wird, dass dauerhafter Friede auf diesem Wege am besten zu erreichen sei (Paris 2004; Bellamy 2004: 19; Richmond/Mitchell 2011: 326). Die Diskussion über den liberal peace ist die zentrale theoretische Debatte über den Gegenstand Intervention: Bliesemann de Guevara/Kühn (2010); Paris (2010); Richmond/Franks (2009); Roberts (2011). 


\subsection{Der erwartbare Widerstand}

Als Bezeichnung von Akteuren des Widerstands in Interventionen hat sich in der wissenschaftlichen Literatur seit einem Artikel von Stephen John Stedman (1997) der wirkmächtige Begriff des »Spoilers« etabliert (Nilsson/Söderberg Kovacs 2011). In der ursprünglichen Bedeutung sind Spoiler Akteure, die ihre Position durch Friedensprozesse gefährdet sehen und mit GewaltFrieden verhindern wollen. Inzwischen wird der Begriff jedoch auch im breiteren politischen Kontext verwendet: Ein Spoiler ist dann derjenige, dersich aus Friedensprozessen zurückzieht und ihnzu behindern droht oder nicht die innere Überzeugung zum Frieden besitzt (Newman/Richmond 2006: 102). Problematisch isthierdieFragenach derDefinitionshoheit:Dain Interventionen wie in Bosnien und im Kosovo Statebuilding und Peacebuilding untrennbar miteinanderverbunden sind, fällt es leicht, Widerstand gegen Interventionspolitik als grundsätzliche Gefahr des Friedensprozesses darzustellen. Externe Akteure tendieren dazu, jede Form des Widerstands als solchermaßen bedrohlich zu delegitimieren (Stewart/ Knaus 2011). Die Literatur verweist jedoch darauf, dass Widerstand in Interventionen kein einheitliches Phänomen ist und verschiedene Formen und Folgen haben kann: Béatrice Pouligny (2006: 218) zeigt beispielsweise, dass für lokale Akteure auch jenseits von Gewalt eine Vielzahl von Formen des Widerstands bestehen, wie beispielsweise das Verzögern oder Ignorieren. Für Oliver Richmond und Audra Mitchell werden solche»critical forms of agency «zu Kritiken des liberalpeace selbst,»upon which local forms of legitimacy may be built « (Richmond/Mitchell 2011: 327). Hier dient Widerstand nicht nur der Delegitimierung von Intervention, sondern auch der Konstituierung von Alternativen und kann eine integrative Wirkung innerhalb der intervenierten Gesellschaften entfalten (vgl. dazu auch Mac Ginty 2011).

Der Begriff des politischen Widerstands, den wir verwenden, versucht die Unerreichbarkeit der Intervenierenden aus Perspektive der Intervenierten in politischen Konflikten einzufangen: Der Begriff Opposition scheint uns für diese Beziehung nicht angebracht, da Opposition sich zumeist auf ein Gegenüber innerhalb des politischen Systems bezieht, also z. B. auf eine Regierung (Luhmann 1989). Die Intervenierenden sind jedoch keine Regierung, sie positionieren sich außerhalb oder oberhalb der polity. Opposition hat ultimativ das Ziel, die Regierung zu ersetzen, also selbst diese Position einzunehmen (Albrecht 2009) - dies ist im Falle der Intervention unmöglich.

Doch auch der Begriff des Widerstands bedarf einer Eingrenzung: Wir bezeichnen ihn als politisch, weil er sich nicht zwingend außerhalb der politischen Institutionen manifestiert, gewaltbehaftet ist oder das politische System revolutionieren will. Der politische Widerstand ist für uns vor allem durch seinen relationalen Cha- 
rakter zur Intervention gekennzeichnet, analog der relationalen Konzeption von Widerstand zu Macht bei Michel Foucault: Der politische Widerstand ist »die andere Seite, das nicht wegzudenkende Gegenüber« (Foucault 1977: 117) der Intervention. Er kann nicht losgelöst von ihr analysiert werden, da er nur in Beziehung zu ihr entsteht und Sinn erhält, wie wir in den Fallanalysen zeigen wollen.

Unser Begriff von Widerstand ist also kein normativer. Wir vermuten weniger einen legitimen - im Sinne von berechtigt -, sondern einen erwartbaren politischen Widerstand, der seine Kraft aus dem fehlenden Herrschaftsvertrag zieht: Politischer Widerstand ist für uns Handeln (in Sprache und Aktion und unter Verzicht auf bewaffnete Gewalt), welches auf die Fremdheit, fehlende Legitimation und Rechenschaftspflicht der Intervenierenden verweist und eigene Positionen in Ablehnung dazu entwickelt. Widerstand ist insofern als erwartbare Folge von Intervention zu verstehen, er ist die andere Seite der Medaille. Seine Wirkungen müssen in den jeweiligen Fällen bewertet und können nicht von vornherein als negativ deklariert werden.

Im Folgenden sollen zwei Träger politischen Widerstands vorgestellt werden: Zunächst Widerstand in politischen Institutionen am Beispiel der Partei Savez Nezavisnih Socijaldemokrata (SNSD) in Bosnien (Kapitel 3), dann außerinstitutioneller, aktionistischer Widerstand am Beispiel der Bewegung VETËVENDOSJE! im Kosovo (Kapitel 4). Ziel ist es, mithilfe der empirischen Erkundung der beiden so unterschiedlichen Organisationen die Beziehung zwischen Intervention und Widerstand aufzuzeigen. Dafür wird wie folgt vorgegangen: Erstens stellen wir jeweils für die beiden Fälle Bosnien und Kosovo die konkreten Varianten des fehlenden Herrschaftsvertrags dar. Zweitens zeichnen wir die Entwicklung der Organisationen nach und klären drittens, wie SNSD und VETËVENDOSJE! sich argumentativ auf die Intervention beziehen, um ihren Widerstand zu begründen. In einem vierten Abschnitt stellen wir jeweils die unterschiedlichen Formen des Widerstands gegen Intervention dar, wobei der SNSD ein Beispiel für Widerstand unter Nutzung staatlicher Kanäle ist, während VETËVENDOSJE! vor allem durch Aktion jenseits der staatlichen Institutionen agiert. Fünftens widmen wir uns den kritischen Reaktionen der Intervenierenden auf Widerstand. Abschließend fragen wir nach der Wirkung des Widerstands innerhalb der beiden Gesellschaften. Dabei ist zu beachten, dass ethnische Teilungen in beiden Gesellschaften eine große Rolle spielen. In beiden Fällen hat Widerstand in Bezug auf die Teilgruppen integrativen Charakter, wirkt in Bezug auf die gesamte Gesellschaft jedoch klar polarisierend. Die gemeinsame Bewertung beider Fälle in Bezug auf die Argumentation des Artikels erfolgt abschließend im Fazit (Kapitel 5). 


\section{Institutioneller Widerstand in Bosnien: Der Savez, Nezavisnih Socijaldemokrata (SNSD)}

Als Regierungspartei der serbisch dominierten Entität Bosnien-Herzegowinas (Republika Srpska, Serbische Republik, RS) nutzt der SNSD institutionelle Kanäle zum Widerstand gegen die Intervention. Ziel ist dabei, die Autonomie der RS visà-vis dem Zentralstaat zu bewahren oder auszubauen.

\subsection{Der fehlende Herrschaftsvertrag: Ethnische Machtteilung und Internationales Quasi-Protektorat in Bosnien}

Zentrale Frage der Friedensverhandlungen in Dayton Ende 1995 und des vorangegangenen Krieges war, ob und in welcher Form Bosnien als Staat verfasst sein sollte (Bose 2002). Politische Vertreter der Bosniaken als der größten ethnischen Gruppe präferierten einen integrierten Gesamtstaat. Die bosnischen Serben und bosnischen Kroaten ${ }^{5}$ bestanden auf größtmögliche Autonomie und wollten sich die Möglichkeit einer späteren Vereinigung mit Serbien respektive Kroatien offen halten (Parish 2007). Resultat der Verhandlungen war eine Kompromisslösung, die Bosnien als Staat bestehen ließ, aber weitreichende Mechanismen ethnischer Machtteilung vorsah. Bosnien wurde in zwei Entitäten geteilt, eine bosniakisch-kroatische Föderation und eine serbische Republik, denen der überwiegende Teil staatlicher Kompetenzen übertragen wurde. Zusammengehalten werden beide von zentralstaatlichen Organen mit geringen Kompetenzen.

Ethnische Konfliktlinien um die Verfasstheit des bosnischen Staates bestimmen bis heute das politische Geschehen (Džihić 2009). Diese Auseinandersetzungen haben seit 2006 wieder an Schärfe zugenommen (vgl. Abschnitt 2.3). Die politische Krise soll hier jedoch nicht ausführlich behandelt werden. ${ }^{6}$ Wir konzentrieren uns auf die Auseinandersetzungen zwischen den Intervenierenden (vor allem dem Büro des Hohen Repräsentanten) und dem SNSD seit 2006 als Variante des Widerstands.

Zur Umsetzung des Abkommens von Dayton wurde eine internationale Mission ins Leben gerufen. Die zivile Umsetzung liegt federführend beim Büro des Hohen Repräsentanten (Office of the High Representative, OHR); daneben ist eine Vielzahl anderer Organisationen (etwa OSZE, NATO, EU, Europarat) für Teilaspekte zuständig. Mit der Einführung der sogenannten Bonn Powers hat das OHR 1997 de facto die Befugnisse einer Interimsadministration erhalten. Der Hohe Repräsentant

5 Gemeint sind die ethnisch definierten politischen Eliten, nicht die Bevölkerungsgruppen im Ganzen.

$6 \mathrm{Zu}$ Ursachen und Komplexität der aktuellen politischen Krise in Bosnien vgl. Bassuener/Weber (2010) und Džihić (2009). 
kann seitdem Gesetze erlassen und Personen des Amtes entheben. Er ist dem eigens geschaffenen Friedensimplementierungsrat (Peace Implementation Council, PIC) und dessen Steuerungsgremium (Peace Implementation Council Steering Board, PIC Steering Board) gegenüber rechenschaftspflichtig. ${ }^{7}$ Der bosnischen Bevölkerung und Regierung gegenüber ist der Hohe Repräsentant kaum rechenschaftspflichtig (Venice Commission 2005: 2). Besonders bedenklich ist dabei, dass auch kein transparentes Verfahren und keine Berufungsinstanz für durch den Hohen Repräsentanten abgesetzte Amtsträger existieren (Venice Commission 2005: 23). In Bezug auf die politischen Ziele und Vorhaben der Intervention werden die bosnischen Vertreter meist nur zu Konsultationen herangezogen, wobei die bosnischen Vertreter kein Stimmrecht haben und abhängig von der Tagesordnung entschieden wird, wer zu welcher Sitzung eingeladen wird. ${ }^{8}$ Zwar gibt es eine Ombudsperson mit beratendem Charakter, die Menschenrechtsverletzungen der Intervenierenden nachgeht (Zaum 2007; Chesterman 2004) und seit November 2000 existiert die Möglichkeit, Gesetze des Hohen Repräsentanten vor dem bosnischen Verfassungsgericht auf ihre Verfassungsmäßigkeit hin überprüfen zu lassen. Beides berührt aber den grundlegenden Mangel an Rechenschaftspflicht und Erreichbarkeit nicht.

Zielte die Politik der Intervenierenden anfangs vor allem auf die Umsetzung des Friedensabkommens, so änderte sich der Fokus spätestens mit dem Amtsantritt Paddy Ashdowns als Hoher Repräsentant im Frühjahr 2002. Dayton und seine rigiden power-sharing-Mechanismen wurden verstärkt als Hindernis für stabile Staatlichkeit gesehen (Ashdown 2002). Die Intervenierenden konzentrierten sich nun vor allem darauf, den Zentralstaat gegenüber den Entitäten zu stärken (Gromes 2007). Eine Verteidigungsreform und die (erfolglose) Polizeireform zielten darauf ab, mehr Kompetenzen auf Staatsebene anzusiedeln (Lindvall 2009). Darüber hinaus wurden 2006 und dann noch einmal 2009 seitens der Intervenierenden weitere Verhandlungen über eine Verfassungsreform angestoßen, die direkt die verfassungsrechtlichen Grundlagen ethnischer Machtteilung in Bosnien ins Visier nahmen. ${ }^{9}$ Diese Politik der stärkeren Zentralisierung widerspricht vor allem den Interessen der RS. Insofern reagierte die Führung der RS ihrerseits mit einem Politikwechsel. War sie früher bemüht, die Implementierung des Abkommens von Dayton

7 Dem PIC gehören 55 Staaten und internationale Organisationen an (PIC 2012). Das vollständige PIC hat sich 2000 das letzte Mal getroffen, sodass das eigentlich relevante Gremium heute das PIC Steering Board ist. Dieses besteht aus Kanada, Frankreich, Deutschland, Italien, Japan, Russland, Großbritannien, der EU-Ratspräsidentschaft, der Europäischen Kommission und der Organisation Islamischer Staaten (OIC), vertreten durch die Türkei.

8 Persönliches Interview mit Barkin Kayaoglu, OHR, am 02.06.2010 in Sarajevo.

9 Beide Male sind die Intervenierenden damit gescheitert (Borić 2010; Hays/Crosby 2006; Sebastian 2007). 
zu verzögern oder zu verhindern, so wurde das Beharren auf Dayton spätestens ab 2006 zentrales Element der Argumentation.

\subsection{Der SNSD}

Der SNSD und insbesondere Milorad Dodik bestimmen seit 2006 die Politik der RS. Galt die Partei lange als moderat und den Intervenierenden gegenüber kooperativ, so änderte sich dies ab 2006. Die Politik des SNSD ist seitdem gegenüber den anderen ethnischen Gruppen konfrontativ und baut gegenüber den Intervierenden auf Widerstand.

Der SNSD wurde 1996 von Dodik gegründet. Eine kurze erste Phase mit Regierungsverantwortung von 1998 bis 2001 war für den SNSD wenig erfolgreich (ESI 1999; ICG 2003). Die Partei gewann aber schnell wieder an Popularität. Sowohl unter der bosnisch-serbischen Bevölkerung wie auch unter den Intervenierenden wurde der SNSD als Alternative gegenüber dem während und seit dem Krieg dominierenden SDS (Serbische Demokratische Partei) wahrgenommen (Gavrić/Banović 2007: 57). Der SNSD beschreibt sich als sozialdemokratisch, analog zu den meisten Parteien Bosniens hat das politische Programm abseits ethnisch definierter Politik aber wenig Kontur. Die Partei ist intern ausgesprochen hierarchisch organisiert, mit Dodik unangefochten an der Spitze. ${ }^{10}$ Die Partei übernahm die Regierungsgeschäfte in der RS im Frühjahr 2006 im Zuge eines erfolgreichen Misstrauensvotums gegen Regierungschef Dragan Čavić (SDS) (Gavrić/Banović 2007; Gromes 2007) und regiert seit den Wahlen im Oktober 2006 als erste Partei mit absoluter Mehrheit.

Der Aufstieg des SNSD ging Hand in Hand mit einer Reihe von Entwicklungen, die das politische Klima in Bosnien drastisch verschlechterten. Der gescheiterte Versuch einer Verfassungsreform im Frühjahr 2006 stellte den Minimalkonsens von Dayton wieder in Frage. Die Reform wurde vor allem von der bosniakischen Partei SBiH (Partei für Bosnien und Herzegowina) und ihrem Vorsitzenden Haris Silajdžić kritisiert, denen die Änderungen nicht weit genug gingen (Sebastian 2007). Der ethnische Minimalkonsens war nun allerdings wieder Verhandlungsthema, was das ohnehin fragile Vertrauen unter den bosnischen Parteien weiter unterminierte. Im folgenden Wahlkampf für die Wahlen im Herbst 2006 dominierte nationalistische Rhetorik. Dodik forderte etwa für die RS das Recht, ein Unabhängigkeitsreferendum abzuhalten, während Silajdžić unter dem Slogan »100\% $\mathrm{BiH}$ « die Ab- 
schaffung der Entitäten einforderte (Gavrić/Banović 2007). Diese Rhetorik zahlte sich aus: SNSD und SBiH gewannen deutlich an Stimmen hinzu. ${ }^{11}$

Neben der nationalistischen Rhetorik zeichnet sich die Argumentation des SNSD auch dadurch aus, dass die Politik und die Legitimität der Intervenierenden und vor allem des OHR offen in Frage gestellt werden. Es kann davon ausgegangen werden, dass auch dies zur Popularität Dodiks beigetragen hat. Erstens ist die Zustimmung zur Politik des OHR unter der Bevölkerung der RS vor allem seit 2004 niedrig. ${ }^{12}$ Und zweitens deutet der Zeitpunkt besonders scharfer Attacken auf das OHR 2006 und 2010 vor den jeweiligen Wahlen darauf hin, dass Dodik sich diesen Unmut gezielt zunutze machen wollte. ${ }^{13}$ Dieser Widerstand ist deshalb auch durchaus opportunistisch, sein Anliegen ist der Machterhalt. Widerstand gegen die Intervention nützt hierbei einerseits zur Mobilisierung von Wählern. Andererseits stellen die Intervenierenden und ihre Bemühungen, den Zentralstaat zu stärken, aus Sicht des SNSD eine Bedrohung serbischer Autonomie in Bosnien dar.

\subsection{Argumentation in Bezug auf externe Herrschaft}

Das zentrale Thema des SNSD ist der Schutz der Autonomie der RS. Das Vokabular schließt dabei an den Diskurs der Intervenierenden an. Beispielsweise sind Europa und Europäisierung zentrale Kategorien, vor deren Hintergrund der SNSD sein eigenes, ethnopolitisches Programm formuliert. Es wird argumentiert, eine EU-Integration erfordere Gleichberechtigung innerhalb des bosnischen Staates. Damit ist hier gemeint, dass die bosniakischen Vertreter den Staat nicht dominieren sollten (Džihić 2007: 11). Dayton wird als ein Instrument des Schutzes vor Diskriminierung konstruiert. In Bezug auf die Intervention wird betont, dass nachhaltige Lösungen nur aus eigener Kraft entstehen könnten. Im Parteiprogramm des SNSD liest sich das etwa wie folgt:

11 Die Stimmen für das gesamtbosnische Repräsentantenhaus werden auf Entitätsebene ausgezählt: Der SNSD steigerte sich von 22,39\% in der RS und 0,81\% in der Föderation im Jahr 2002 auf $46,93 \%$ in der RS und $0,85 \%$ in der Föderation im Jahr 2006. SBiH verbesserte die Ergebnisse von $16,19 \%$ in der Föderation und 3,9\% in der RS in 2002 auf 22,99\% in der Föderation und 4,16\% Prozent in der RS in 2006 (Izborna Komisija Bosna i Hercegovina 2002 a; 2002 b; 2006).

12 UNDP hat hierzu bis 2008 vierteljährliche Umfragen im Rahmen eines Frühwarnsystems in Auftrag gegeben. Zwischen 2002 und 2004 lagen die Zustimmungswerte zum OHR in Gegenden mit bosnisch-serbischer Mehrheitsbevölkerung meist zwischen 40\% und 50\%. Im Sommer 2004 fielen diese Werte auf zwischen 30\% und 40\%, in den letzten beiden Quartalen in 2007 fielen sie weiter auf knapp unter 30\% und stiegen 2008 wieder auf knapp über 30\% (UNDP BiH 2006; 2007; 2008).

13 Persönliches Interview mit Christian Schwarz-Schilling am 23.11.2012 in Berlin. 
»Das Friedensabkommen ist das Minimum politischen Konsenses in $\mathrm{BiH}$, es ist aber auch ein internationales Abkommen, das unter ständigem Beschuss von politischen Kräften steht, die mit dem Ende des Krieges ihre politischen Ziele nicht erreicht haben. Seine inhaltliche Revision würde zu neuen Spannungen und Konflikten in der ganzen Region führen. Der Friedensvertrag und der Verfassungsrahmen können nur durch Konsens und wirklichen Willen der Entitäten, konstituierenden Völker und Bürger Bosniens geändert werden, und nur hin zu einem effizienten, diskriminierungsfreien System im Einklang mit europäischen Standards. Jeder Versuch, ein Mehrheitsprinzip einzuführen oder Lösungen von außen aufzuoktroyieren wird bloß eine Atmosphäre des Misstrauens schaffen und BiH eine unsichere und instabile Zukunft bescheren $\ll\left(\right.$ SNSD o.J.). ${ }^{14}$

Im Widerstand gegen die Intervenierenden und OHR gibt es zwei zentrale Argumentationslinien. Erstens werden die vom OHR angestoßenen Reformvorhaben kritisiert, insbesondere insofern sie eine stärkere Zentralisierung zum Inhalt haben. Gordan Milošević, Dodiks außenpolitischer Berater, erklärt so auch die Forderungen des RS-Premiers ${ }^{15}$ nach einem Unabhängigkeitsreferendum:

»He says, please, respect Dayton, let's make improvements, but on the Dayton structure, let's not touch the basic elements of balance that were established in Dayton because they are essential for this country. They are essential for our feeling of safety in this country. Let's move forward, let's do practical requirements [for the EU integration process], let's fulfill them, but let's not change the basics of our structure. But if someone insists on changing this structure, and in undermining or completely abolishing our autonomy, then one has to understand, leaving Dayton means that all options are open. Not only a centralized state. But secession, also. Like, if Dayton is not an option anymore, how comes that a centralized state is a legitimate option and secession is not, that's what he asks, that's what he says. 16 $^{16}$

Die zweite Argumentationslinie bezieht sich auf die Präsenz und die Befugnisse des OHR an sich. Hier folgt die Argumentation im Kern dem schon von Knaus und Martin (2003) vorgebrachten Argument, die Befugnisse des OHR begünstigten eine kompromisslose nationalistische Politik, weil schlicht keine Notwendigkeit für Kompromisslösungen bestünde, solange Gesetze letztendlich ohnehin durch das

14 Übersetzt von Sarah Riese.

15 Seit den Wahlen 2010 ist Dodik Präsident der RS.

16 Persönliches Interview mit Gordan Milošević, SNSD, am 24.05.2010 in Banja Luka. 
OHR erlassen werden. Nina Sajić, außenpolitische Beraterin des serbischen Präsidentschaftsmitglieds ${ }^{17}$ Nebojša Radmanović, bringt das auf den Punkt:

»The International Community did play an important role in bringing us to the same table, but failed to see the point to draw back. As long as there is someone you can go and complain to, it's going to be very difficult to reach an agreement. Because we all start from very maximalist positions. Instead of reaching for the win-win situation, we always try to have a 'win-zero' situation, which is very detrimental. $\ll^{18}$

Daneben wird vor allem auch die fehlende Rechenschaftspflicht und der undemokratische Regierungsstil des OHR kritisiert. Ein Beispiel ist ein Bericht an den UNSicherheitsrat von Mai 2011. Der Bericht befasst sich mit einem zu dieser Zeit geplanten Referendum ${ }^{19}$ in der RS, in dem darüber abgestimmt werden sollte, ob die Bürger der RS vom OHR erlassenen Gesetzen vor allem in Bezug auf gesamtstaatliche Gerichte zustimmen:

»The international community can play a beneficial role in its relations with $\mathrm{BiH}$, but only if members of the international community respect and defend the rule of law and constitutional democracy, including with regard to their own actions and actions of the High Representative. [...] Questioning actions of the High Representative and seeking the views of our citizens are not violations of the Dayton Accords, but are means of exercising democracy and legally protected rights« (Office of the Prime Minister 2011: 3-4).

Dem Beharren auf demokratische Prinzipien auf internationalem Parkett steht eine deutlich abweichende Regierungspraxis gegenüber. Erstens ist das politische System durch informelle Netzwerke gekennzeichnet, die Dodik eine umfassende Kontrolle des politischen und wirtschaftlichen Lebens ermöglichen (Džihić 2009). Zweitens wird die Meinungsfreiheit zunehmend eingeschränkt (Pilsel 2009).

17 Bosnien hat nicht einen Präsidenten, sondern eine aus drei Mitgliedern (bosniakischer, kroatischer und serbischer Herkunft) bestehende Präsidentschaft. Radmanović ist das serbische Mitglied, Frau Sajić arbeitete zum Zeitpunkt des Interviews in seinem Büro.

18 Persönliches Interview mit Nina Sajić, SNSD, am 24.05.2010 in Banja Luka.

19 Dieses geplante Referendum wurde seitens der Intervenierenden als Frontalangriff sowohl auf das OHR als auch auf eine unabhängige bosnische Justiz gewertet (ICG 2011 a; Kovačević 2011). Insofern versuchten die Intervenierenden auf unterschiedlichen Wegen das Referendum zu unterbinden. Erfolgreich war letztendlich eine EU-Initiative, die einen Dialog über Reformen im Justizwesen versprach (ICG 2011 b). 


\subsection{Formen des Widerstands}

Der SNSD als Regierungspartei agiert in Bezug auf die Intervention vor allem innerhalb staatlicher Institutionen und in für einzelne Reformvorhaben geschaffenen Kommissionen. Damit bewegt sich auch ein Großteil der Formen des Widerstands innerhalb dieser Strukturen.

Beispielsweise wird in politischen Konflikten häufig verfassungsrechtlich argumentiert. Da das OHR auf Basis der bosnischen Verfassung agiert, bietet sich diese als Argumentationsgrundlage an. Ein Beispiel hierfür war die gescheiterte Polizeireform. Für die gesamtstaatliche Polizeistruktur wäre eine Verfassungsänderung notwendig gewesen, da hier die Zuständigkeit klar auf Entitätsebene liegt (Donais 2006). Die Unterhändler der RS argumentierten also, die Polizeireform sei eigentlich der Versuch einer Verfassungsreform durch die Hintertür und sie hätten rechtlich keine Befugnisse, dem zuzustimmen. ${ }^{20}$

Zweitens sind (angedrohte) Blockaden zentralstaatlicher Institutionen in handfesten Konfrontationen ein probates Mittel. Diese Strategie wurde auch zu Zeiten des SDS angewandt (ICG 2005: 8). Erneut kam es 2009 zu der Drohung, dieses Mal kollektiv die zentralstaatlichen Organe zu verlassen. Aufgrund ethnischer Proporzregelungen wären die zentralstaatlichen Organe dann nicht arbeitsfähig.

Drittens spielt, wie oben schon erwähnt, das internationale Parkett und eine Sprache, die dort gehört, verstanden und für legitim befunden wird, eine zentrale Rolle. Hierin unterscheidet sich der SNSD von seinen Vorgängern: Die Vertreter der RS sprechen heute Peacebuilding-Sprache. Besonders deutlich wird dies am schon zitierten Bericht an den UN-Sicherheitsrat (Office of the Prime Minister 2011). Die zentralen Kategorien sind nicht serbische Autonomiebestrebungen, sondern Demokratie und Menschenrechte. Aufschlussreich ist auch ein Blick auf die Literaturverweise. Mit unterschiedlichen Berichten der International Crisis Group, dem »European Raj« von Gerald Knaus und Felix Martin (2003) oder den Balkan Crisis Reports des Institute for War and Peace Reporting werden dieselben Quellen zitiert, derer sich internationale Beobachter auch bedienen. Auch darüber hinaus ist die RS unter Dodik ein Beispiel für die Internationalisierung des Interventionsstaates (Bliesemann de Guevara 2008). Wenn international definiert wird, was und wie der Staat zu sein hat, so wenden sich intervenierte Akteure ihrerseits an ein internationales Publikum. Vertreter der RS fallen durch professionelles Auftreten gegenüber Internationalen auf, inklusive auf Englisch verfassten Kurzübersichten über aktuelle politische Standpunkte der Entitätsregierung. Zudem wenden sie sich auch gezielt 
an die Dienstherren der Intervenierenden. Neben dem erwähnten Bericht an den UN-Sicherheitsrat versucht vor allem die RS mittels Lobbying in Washington Einfluss auf die Politik zu nehmen. ${ }^{21}$

\subsection{Reaktionen}

Die Intervenierenden tun sich in ihren Reaktionen auf den SNSD schwer. Sie sind sich darin auch mitnichten einig. Obwohl die Rhetorik des SNSD schon im Wahlkampf auf nationalistische Töne einschwenkte, waren die Beurteilungen der Intervenierenden damals durchaus noch positiv. Einerseits war der SNSD nicht mit Kriegsverbrechen in Verbindung zu bringen und andererseits stimmten die wirtschaftlichen Erfolge nach der Amtsübernahme Dodiks hoffnungsvoll (Gavrić/Banović 2007: 62). ${ }^{22}$ Es war aber schnell klar, dass Dodik zu Kooperation mit dem OHR kaum bereit sein würde (Loza 2007). Schon 2006 und seitdem immer wieder wurde die Absetzung Dodiks diskutiert. Dass das OHR davor zurückschreckte, lag einerseits an mangelnder internationaler Unterstützung, und andererseits daran, dass nicht ausgemacht war, dass Dodik dieser Order Folge leisten würde. Schon 2006 entgegnete er Drohungen des damaligen Hohen Repräsentanten Christian SchwarzSchilling:

»If the High Representative wants to see that I can gather 200,000 people in Banja Luka, he can try to remove me, and we shall see what will happen. Do you want me to bring 50,000 Serbs to demonstrate in Sarajevo now? (Globus, 17.01.2007, zitiert nach ICG 2007: 7).

Der Versuch, Dodik abzusetzen, hätte offene Konfrontation bedeutet. Dieses Risiko wollte das OHR nicht eingehen.

\subsection{Wirkung? Verstärkte Autonomie}

Der Konfrontationskurs des SNSD gegenüber dem OHR hat das Internationale Quasi-Protektorat in Bosnien de facto beendet. Dodik hat den Hohen Repräsentanten mehrfach herausgefordert und gedroht, seine Anordnungen zu ignorieren. Das OHR ist in diesen Fällen vor einem offenen Machtkampf zurückgeschreckt. Damit

21 Persönliches Interview mit Kurt Bassuener am 04.05.2010 in Sarajevo; persönliches Interview mit einem Botschaftsmitarbeiter am 11.05.2011 in Sarajevo; Office of the Prime Minister, Republic of Srpska Government (2011).

22 Diese basierten allerdings vornehmlich auf einer umfangreichen Finanzspritze, die der Verkauf der RS Telekom an Telekom Srbija mit sich brachte (ICG 2011 b). Dieses Geschäft fand im Übrigen aber vor dem Amtsantritt Dodiks statt (Falconer-Stout 2009). 
erscheint das OHR heute deutlich weniger mächtig als dies noch vor einigen Jahren der Fall war. Zudem hat die RS die Zusammenarbeit mit dem OHR in weiten Teilen aufgekündigt. ${ }^{23}$ Insofern war die Herausforderung durchaus erfolgreich.

Auf die RS selbst bezogen, hat diese Politik durchaus eine integrative Wirkung. Waren die Loyalitäten auch vor Dodik vor allem auf die RS und nicht auf Bosnien als Ganzes bezogen, so herrscht nun ein neues Selbstbewusstsein. Gesamtbosnisch hat die Politik des SNSD jedoch deutlich polarisierend gewirkt. Im Zusammenspiel mit dem konfrontativen Politikstil, insbesondere von Haris Silajdžić zwischen 2006 und 2010, hatte diese Politik Anteil daran, dass Kompromisse zwischen den Vertretern der verschiedenen ethnischen Gruppen heute kaum noch möglich erscheinen.

Zusammenfassend lässt sich festhalten, dass Widerstand hier vor allem auf den eigenen Machterhalt zielt und darin durchaus erfolgreich ist. Die Argumentation des SNSD offenbart immer wieder den wunden Punkt der Intervenierenden. Ihre mangelnde Rechenschaftspflicht bietet Angriffsfläche. Insofern ist der Widerstand auch Folge des Charakters der Intervention selbst und kann von dieser getrennt nicht bewertet werden.

Diese enge Beziehung zwischen Intervention und Widerstand lässt sich auch in einem weiteren Fall, der Bewegung VETËVENDOSJE! im Kosovo, zeigen.

\section{Aktionistischer Widerstand im Kosovo: Die Lëvizja VETËVENDOSJE! (Bewegung Selbstbestimmung)}

"Self-determination! Without condition. Until the final separation. Until the complete liberation of our country« (Vetëvendosje! $2010 \mathrm{~d}$ ).

\subsection{Der fehlende Herrschaftsvertrag: Interventionen im Kosovo seit 1999}

Ein zusammenfassender Überblick über Governance im Kosovo seit 1999 erweist sich als kompliziert. Am Beginn steht die Internationale Administration seit Sommer 1999, autorisiert durch die Sicherheitsratsresolution 1244. Deren international mandatierte Autorität war de jure unbegrenzt und der Sondergesandte des UN-Generalsekretärs tatsächlich »the sole legislative and executive authority in Kosovo« (Brand 2003: 8). Dieses Mandat traf auf de facto vorhandene parallele Herrschaftsstrukturen, hauptsächlich bestehend aus einer provisorischen Regierung verschiedener UÇK-Führer (Ushtria Çlirimtare e Kosovës, Befreiungsarmee des Kosovo)

23 Gruppeninterview mit Mitarbeitern der EU Integration Unit der RS am 24.05.2010 in Banja Luka; persönliches Interview mit Nina Sajić, SNSD, am 24.05.2010 in Banja Luka. 
und der Exil-Regierung der Republik Kosovo unter der Führung von Ibrahim Rugova (vgl. Brand 2003: 11-13).

Die Internationale Administration, in sich selbst vielgestaltig und begleitet von der NATO Kosovo Force (KFOR), begann offizielle Foren für kosovarische politische Akteure zu gründen, zunächst noch 1999 den Kosovo Transitional Council, ab 2000 dann die Joint Interim Administration Structure (JIAS). 2000 begann parallel der Wahlprozess, zunächst auf kommunaler Ebene. Im Frühjahr 2001 wurde dann ein Constitutional Framework for Provisional Self-Government erlassen. Er wurde Grundlage der Wahlen ab Herbst 2001 und der Provisional Institutions of Self-Government (PISG) zwischen 2002 und Anfang 2008. In den knapp zehn Jahren seit 1999 wurde die Regierungsbeteiligung und legislative Tätigkeit der Kosovaren stetig ausgebaut. Bis 2008 galt jedoch noch der absolute Vorbehalt der United Nations Mission in Kosovo (UNMIK) in exekutiven, legislativen und judikativen Angelegenheiten - trotz mehrerer nationaler Wahlen. Auch seit der Unabhängigkeitserklärung vom 17. Februar 2008 ist die Souveränität der Republik Kosovo weiterhin durch externe Einflussmöglichkeiten und Vorbehalte eingeschränkt (Briscoe/Price 2011). Zwar endete im September 2012 die supervised independence des Kosovo unter Aufsicht des International Civilian Representative (ausgestattet mit exekutiven Befugnissen), zuständig für die Implementierung des Ahtisaari-Plans, weiterhin werden aber Missionen wie die Rechtstaatsmission der EU (EULEX), die KFOR, die OSCE-Mission im Kosovo (OMIK) und UNMIK präsent bleiben - als »UNSECR 1244 Legacy in Post Independence Kosovo« (Kallaba/Ferati 2012).

In der Rückschau auf die Intervention seit 1999 muss konstatiert werden, dass die Distanz zwischen externen und internen Akteuren besonders in Fragen der Rechenschaftspflicht und Verantwortlichkeit der Internationalen ausgeprägt war. Während von Beginn der Mission an für Internationale, bis auf schwerwiegende Anschuldigungen, Immunität galt (Rawski 2002), wurden zwar später vielfältige Mechanismen der »Accountability« eingeführt (Human Rights Watch 2007: 13-27), eine erfolgreiche Praxis hat sich aber nie entwickelt. Visoka (2012) spricht deshalb auch von der »Kafkaesque Accountability« der internationalen Governance.

\subsection{Die Lëvizja VETËVENDOSJE!}

Die Bewegung VETËVENDOSJE! ist ein Produkt der Intervention. Sie war keine politische Kraft oder Konfliktpartei in den 1990er Jahren. Zwar hat die Bewegung ihre Wurzeln im Kosovo Action Network (KAN), das bereits Ende der 1990er Jahre in kleinerem Umfang aktiv war, Startpunkt für die Mobilisierung der Bewegung war aber erst die internationale Entscheidung im Sommer 2005, den zukünftigen 
Status des Kosovo zusammen mit Serbien zu verhandeln, was die Bewegung strikt ablehnte (Kurti 2011: 93). Eindrücklich ist die Metamorphose in nur fünf Jahren von einer aktionistischen Widerstandsbewegung hin zu einer politischen Partei, die seit 2010 im Parlament auf Basis von 12,69\% der Wählerstimmen mit 14 Sitzen vertreten ist (KIPRED 2011: 19-22).

Zentral für die Bewegung ist die Person Albin Kurti. Seine Bedeutung liegt nicht nur in politischen Fähigkeiten begründet, er selbst ist ein Symbol von Widerstandshandlungen: Sei es seine Verhaftung bei einer Großdemonstration am 10. Februar 2007, als UNMIK-Polizisten auf einer Großdemonstration in Pristina zwei Demonstranten durch Einsatz von Gummigeschossen töteten ${ }^{24}$, seine Weigerung, die Legitimität des Prozesses gegen sich anzuerkennen (Kurti 2010), oder seine erneute Verhaftung im Juni 2010 und Verurteilung von einem international und national besetzten Gericht zu neun Monaten Haft - diese Ereignisse sind starke Narrative, die er nutzt, um die Notwendigkeit von Widerstand zu begründen (Kurti 2011).

\subsection{Argumentation in Bezug auf externe Herrschaft}

So vielfältig die Themen in der Programmatik sind (Vetëvendosje! 2010 a), so eindeutig sind die zentralen programmatischen Ankerpunkte zu erkennen: Freiheit und Selbstbestimmung, wobei die beiden Begriffe auch synonym benutzt werden. Für die Bewegung typisch wird nicht zwischen Freiheit und Selbstbestimmung eines Individuums oder eines Kollektivs (Synonyme hierfür: state, the people) unterschieden, sondern eine untrennbare Verbindung zwischen beiden hergestellt:

»Collective freedom is a fundamental condition for individual freedom. When there is no collective freedom, individual freedom is only an accidental occurrence. Individual freedom can only be realized through total societal freedom. It is because of the lack of collective freedom that the standard of living of our people is falling« (Vetëvendosje! $2010 \mathrm{b:}$ 2).

In dieser Konzeption von Nation und Staat steht die Einheit über den einzelnen Teilen. So erklärt sich auch die strikte Ablehnung jeder Form der von den Intervenierenden immer unterstützten ethnischen Dezentralisierung im Kosovo:

24 In Folge mussten der UNMIK-Polizeichef und der Innenminister des Kosovo zurücktreten, eine UN-Untersuchung beurteilte das Vorgehen der UNMIK-Polizei als fehlerhaft. Anklage gegen Polizisten wurde jedoch keine erhoben (vgl. UNMIK Press Briefing 2007; Dean 2007). 
»Decentralization up to today, based on ethnic principles, has been in the service of daily and disintegrating politics for the territory and society. It is very dangerous also for the future of the state of Kosova« (Vetëvendosje! $2010 \mathrm{a}: 6$ ).

Die Bewegung konstruiert die Geschichte des Kosovo als eine Geschichte der Unterdrückung, wobei die Bevölkerung des Kosovo als Opfer implizit mit den Kosovoalbanern gleichgesetzt wird. Unterdrücker im 20. Jahrhundert und bis in die Gegenwart ist dabei Serbien [»Serbia's intellectual and political elites«, »government of Serbia« (Vetëvendosje! 2010 b: 2)] - wobei nicht das »serbische Volk«, sondern die politische Elite als Gegner konstituiert wird. Verhandlungen mit Serbien über den Status des Kosovo wurden und werden strikt abgelehnt: Diese Ablehnung ist bis in die Gegenwart Grund für Proteste, zuletzt über die Bezeichnung des Kosovo in regionalen Verhandlungsrunden oder über die neu aufgenommenen Verhandlungsrunden seit Herbst 2012 zwischen Pristina und Belgrad.

Die Intervention im Kosovo seit 1999 und die Resolution 1244 werden als eine Fortführung der historischen Unterdrückung verstanden und verhindern echte Selbstbestimmung und Freiheit. Dementsprechend fällt auch die Einschätzung der UNMIK aus: »non democratic regime« (Vetëvendosje! 2010 b: 2), »colonial occupation« und »absolute ruler« (Vetëvendosje! 2006). Die Kritik schließt auch »various international and supranational organizations « (Vetëvendosje! $2010 \mathrm{a}: 1$ ) ein. Deren Herrschaft soll besonders deshalb beendet werden, weil sie nicht gewählt und nicht rechenschaftspflichtig ist (Vetëvendosje! $2010 \mathrm{a:} \mathrm{3).}$

Aber nicht nur den Intervenierenden, sondern auch den nationalen Institutionen ist Widerstand zu leisten. Den vormaligen PISG (bis 2008) wurde die kosovarische Identität abgesprochen ${ }^{25}$, und trotz Wahlen waren sie »not rightful representatives of the people's interest, because the fundamental interest of the people is the realization of its will« (Vetëvendosje! 2010 b: 2). Die Kritik an der Regierung hält auch nach der Unabhängigkeitserklärung 2008 - für die Bewegung keine echte Unabhängigkeit aufgrund der anhaltenden internationalen Präsenz - an. Die Regierung sei korrupt, international abhängig, autoritär, undemokratisch und verrate die Interessen der kosovarischen Bevölkerung (Vetëvendosje! 2012; Vetëvendosje! 2010 c: 1$)$.

Die engen Beziehungen zwischen den nationalen politischen Eliten und den Internationalen halten die Unfreiheit und fehlende Selbstbestimmung auch im Alltag aufrecht:

$25 »$ Kosovar institutions without sovereignty are, in fact, neither Kosovar nor real institutions [...]« (Vetëvendosje! 2006). 
"Years of dwelling in Kosova have now turned thousands of internationals into 'local internationals', a different species from their compatriots back home. Years of international rule have turned local politicians and NGOs into 'international locals', a species that differs from their compatriots in Kosova. The 'local internationals' and the 'international locals' are kept together by a happy marriage of interest, providing the system's internal cohesion « (Kurti 2011: 92).

Die kompromisslose und radikal-kritische Sprache der Bewegung spiegelt sich in den Aktionen der Bewegung wider.

\subsection{Formen des Widerstands}

Öffentliche Kommunikation ist zentral. VETËVENDOSJE! verfügt über eine professionelle Kommunikationsstrategie: Es werden regelmäßig Roundtables und Informationsveranstaltungen abgehalten, auf der Website sind tägliche und wöchentliche Newsletter und Informationen über Aktionen, die wichtigsten programmatischen Papiere und auch alle internationalen oder nationalen Dokumente, auf die sich die Kritik der Bewegung richtet, zu finden. Adressaten der politischen Kommunikation sind ganz eindeutig nicht nur Kosovaren, regelmäßig werden Dokumente auch in Englisch, Französisch und Deutsch veröffentlicht. Die Dokumente richten sich an ein internationales Publikum und verwenden ebenfalls die Begriffe und die Sprache der Intervention - auch wenn sich die Bewegung stärker kritisch von dieser Sprache abgrenzt, anstatt sie nur zu inkorporieren. Teilweise werden kritische Analysen verfasst und über die Website zugänglich gemacht, so z. B. die »Deconstruction of the Declaration of Indepedence« (Vetëvendosje! 2010 e). Auf Facebook hat die Bewegung die Unterstützung von über 166.000 Nutzern (Stand 16. August 2013).

Besonders sichtbare Kommunikation sind die gesprayten Parolen, die im gesamten Kosovo einheitliche Forderungen in einem einheitlichen Design an Hauswänden transportieren. Prominenter Ursprung war der Slogan »Jo Negociata, VETËVENDOSJE! « (Keine Verhandlungen, Selbstbestimmung!) von 2005. Andere Beispiele sind der Slogan »Big Brother is watching you« auf KFOR-Plakaten und 2009 der Spruch »EULEX made in Serbia« oder $2006 » 12.44$ Time's Up - UNMIK Go Home« ${ }^{26} 2007$ wurde nach den Demonstrationen im Februar »Murderers« auf UNMIK-Fahrzeuge geschrieben (Vetëvendosje! $2010 \mathrm{~d}$ : 4-5). 
Obwohl die Bewegung ihre Friedfertigkeit (non-violent) unterstreicht (Kurti 2011: 94), kommt es bei Demonstrationen häufig zu Gewalt. Auch werden regelmäßig Personen mit Lebensmitteln beworfen oder, wie im Sommer 2009, EULEXFahrzeuge zerstört. Als gänzlich gewaltfrei kann die Bewegung deshalb nicht bezeichnet werden, auch wenn es sich nicht um bewaffneten Widerstand handelt.

\subsection{Reaktionen}

Die Reaktionen der internationalen und nationalen Institutionen auf die Bewegung sind eindeutig: Alleine die Zahl der Verhaftungen in den frühen Jahren der Bewegung belegen dies. ${ }^{27}$ Die politischen Reaktionen auf die Bewegung sollen hier exemplarisch anhand der Aktionen der Bewegung hinsichtlich der Verhandlungsrunden zwischen Kosovo und Serbien im Januar 2012 aufgezeigt werden. Die Bewegung hatte mehrfach versucht, Grenzübergänge nach Serbien und damit den Warenaustausch zu blockieren.

Der Ton in offiziellen UN-Dokumenten hat sich entspannt: Ist im Report des Generalsekretärs der UN im Februar 2007 noch von einer radikalen Gruppe mit gewalttätigen Absichten die Rede ${ }^{28}$, so werden fünf Jahre später im Report vom 31. Januar 2012 die Proteste nur noch sachlich erwähnt. ${ }^{29}$ Diese Versachlichung mag auch mit der reduzierten Rolle der UNMIK seit 2008 zusammenhängen.

Politisch wird von anderer Stelle deutlicher kritisiert: Der International Civilian Representative und Leiter des International Civilian Office (ICO), Pieter Faith, verurteilte die geplanten Blockaden und warf der Bewegung unverantwortliches Verhalten und die Beschädigung der internationalen Reputation des Kosovo vor (International Civilian Office 2012). Der deutsche Botschafter im Kosovo, Ernst Reichel, warf der Bewegung hinsichtlich einer Kampagne über den Streit um die Namensverwendung des Kosovo in regionalen Foren »kindergarten politics of children« (Aliu 2012) vor. Die Regierung des Kosovo, stets selbst von der Bewegung scharf kritisiert, unterstellte dieser in einer Reihe von Presseerklärungen im Januar 2012 nicht nur Absicht von Gewalt, sondern auch von Manipulation und dem

27 Verhaftungen nach Selbstangaben: 198 (2005), 322 (2006), 84 (2007) (vgl. hierzu Vetëvendosje! 2010 e).

$28 »$ Vetevendosje again held a protest $[\ldots]$ the intent of which was clearly violent. [...] While there is little mainstream support for the actions of this movement, the continued lack of clarity on Kosovo's status [...] creates a fragile environment which was exploited by radical elements. [...] Vetevendosje and their linkages to other groupings such as the War Veterans' Association remain a cause for concern. Radical groups are likely to continue to exploit any public dissatisfaction« (United Nations Security Council 2007: 2-3).

29 Vgl. hierzu United Nations Security Council (2012: 4). 
Wunsch nach Machtergreifung im Land durch organisierte und gewalttätige Proteste. ${ }^{30}$ Die Konflikte zwischen der Bewegung, der Regierung und den Internationalen sind also im vollen Gange, wie es auch die Auseinandersetzungen hinsichtlich der Wiederaufnahme des kosovarisch-serbischen Dialogs seit Oktober 2012 (Aliu 2012) und im Frühjahr 2013 zeigen.

\subsection{Wirkung? Integration und Exklusion}

Die Bewegung ist im Kosovo die andere Seite der Medaille externer Herrschaft. Weniger zentral ist dabei der programmatische Unterschied zwischen dem demokratischen Liberalismus der Intervention und dem zwischen linkem und rechtsnationalistischem Gedankengut angesiedelten Programm der Bewegung (Visoka 2011: 121), sondern die immer wieder betonte Dichotomie von Fremd- und Selbstbestimmung: Alle inhaltlichen Fragen scheinen im Kern auf diese Dichotomie reduziert.

Dies ist auch in Wirtschaftskampagnen der Bewegung seit September $2011 \mathrm{zu}$ sehen: Angesichts der anhaltenden Auseinandersetzung über den extrem hohen Import von Gütern aus Serbien in den Kosovo wurde dazu aufgerufen, lokale Produkte aus dem Kosovo anstelle von importierter Ware zu kaufen. Ein auffälliges Label mit einem roten Herzen kann auf der im Kosovo hergestellten Ware angebracht werden. Auf dem Label selbst ist die Bewegung nicht genannt. Im August 2013 findet sich auf der Website auch ein Emblem »Plej Shqip« (Kauf Albanisch), eine neue Variante der Kampagne. ${ }^{31}$

So wird die Dichotomie zwischen dem Eigenen und dem Fremden für den Kunden sichtbar gemacht. Dadurch soll die bewusste Entscheidung, die eigene Wirtschaft zu unterstützen, möglich werden. Dies ist die zentrale Nachricht der Kampagne, nicht etwa eine kritische Auseinandersetzung mit der liberalen Marktwirtschaft. Das Wahlergebnis von 2010 zeugt von einer motivierenden und integrativen Wirkung der Bewegung, gerade in urbanen Wahlkreisen ${ }^{32}$, und damit von der Fähigkeit, die etablierte politische Ordnung aufzubrechen und sich als eine Alternative zu den politischen Eliten und deren Netzwerken, die seit den 1990er Jahren oder seit dem Konflikt 1999 dominieren, zu präsentieren. Gerade der letzte Punkt ist eine zentrale Legitimierungsdimension: Die Bewegung, dessen Führungspersonal in den 1970er

30 Office of the Prime Minister (2012a); vgl. auch Office of the Prime Minister (2012 b; 2012 c).

31 Siehe hierfür die Bildergalerie von Vetëvendosje! (www.vetevendosje.org; 16.08.2013).

$32 »$ With 15,899 votes, or $17,93 \%$ of their total votes in 2010, VV [Vetëvendosje!; unsere Anmerk.] is registered as being second largest political entity in Prishtina. This is also true for Gjilan, thus disturbing the established political ordering between PDK and LDK in these cities « (KIPRED 2011: 22). 
und 1980er Jahren geboren wurde, soll als zukünftige dominierende politische Kraft des Landes positioniert werden.

Die Gefahren der Bewegung für ihre politischen Gegner, die Medien, möglicherweise auch für Minderheiten im Land und die regionale Stabilität bei anhaltenden Erfolg und einer möglichen Regierungsbeteiligung in der Zukunft, können nicht eingeschätzt werden. Gëzim Visoka hat jedoch in seiner kritischen Analyse darauf hingewiesen, dass die Sprache und Aktionen der Bewegung gerade für Minderheiten bereits beunruhigende Folgen haben:

»It could be argued that, although Vetevendosje subscribes to the principles of (physical) non-violence, its discoursive and affirmative action can cause physiological violence to minority communities in different instances and through different manifestations« (Visoka 2011: 122-123).

Die Bewegung vertieft laut Visoka die ethnische Teilung des Kosovo und schließt die »subaltern«, also Minderheiten im Kosovo, weiter aus (Visoka 2011: 124).

Die langfristige Wirkung der Bewegung wird sich in den kommenden Wahlen entscheiden: Nach der Transformation in eine Partei muss sie sich an der Prozentzahl der abgegebenen Stimmen messen lassen und kann ihre Legitimation nicht nur aus der Mobilisierung auf der Straße ziehen. Es wird sich noch zeigen, wie sehr die Bewegung durch die langsam abnehmende Präsenz der Intervenierenden an Mobilisierungskraft verliert oder sich weiter transformieren kann.

Der Fall der Bewegung VETËVENDOSJE! zeigt, wie eng die Beziehung zwischen Intervention und Widerstand im Kosovo ist. Im anschließenden Fazit soll ausgehend von den beiden Fällen die Beziehung zwischen Intervention und Widerstand abschließend diskutiert werden.

\section{Fazit: Widerstand und Intervention}

Wir haben argumentiert, dass Interventionen als Aushandlungsprozesse zwischen Intervenierenden und Intervenierten zu verstehen sind. Diese auch konfliktiven Aushandlungen werden seitens der Intervenierenden jedoch häufig nicht eingeplant. Herrschaft beruht in Interventionsgesellschaften also nicht auf einer Beziehung zwischen den Beherrschten und den Herrschenden (wie im gedachten Herrschaftsvertrag), sondern höchstens auf dem Versprechen einer solchen Beziehung in der Zukunft. Für uns wird Widerstand gegen die Intervention unter diesen Umständen erwartbar. Sowohl in Bosnien wie auch im Kosovo lässt sich der fehlende Herrschaftsvertrag exemplarisch aufzeigen. Die Interventionen werden so Ausgangspunkt für den Aufstieg von politischen Gruppen, deren Programmatik und Charakter 
auf Widerstand gegen die Intervention beruht, wie wir am Beispiel des SNSD und VETËVENDOSJE! gezeigt haben. Widerstand gegen die Intervention wurde in beiden Ländern dann zu einem Thema, als die Intervention ihren temporären Charakter weitgehend verloren hatte, Anfangsversprechen enttäuscht wurden und der versprochene Herrschaftsvertrag in weitere Ferne rückte (Talentino 2007).

In ihren Argumentationslinien ist beiden Organisationen der Bezug auf das ethnische/nationale Kollektiv und dessen Selbstbestimmung gemein - das genaue Gegenteil der multiethnischen Agenda der Intervention. In Bosnien beinhaltet dies die Wahrung der Autonomie für die RS, in Bosnien und im Kosovo ein Ende internationaler Aufsicht. Die Kritik an den Intervenierenden ist vergleichbar: Beide prangern ein undemokratisches Verhalten und die mangelnde Rechenschaftspflicht der Internationalen an und verweisen auf die vorgeblich eigenen demokratischen Überzeugungen. ${ }^{33}$

Trotz der unterschiedlichen Ebenen des Widerstands, auf institutioneller (SNSD) wie auch außerinstitutioneller Ebene (VETËVENDOSJE!), sind seine Formen oftmals ähnlich - so der Versuch öffentliche Diskurse anzuregen und sichtbare Aktionen, wie Demonstrationen, durchzuführen, oder die sich explizit auch an internationales Publikum richtende PR-Strategie. Unterschiedlich sind die Ausprägungen und Möglichkeiten: Während am Beispiel VETËVENDOSJE! deutlich wird, dass Fremdherrschaft gänzlich abgelehnt wird und auch kosovarische Institutionen trotz deren demokratischer Legitimation politisch angegriffen werden, kann politischer Widerstand in Bosnien auch aus neuen, durch die Intervention geschaffenen Institutionen heraus durchgeführt werden. Dabei werden zumindest grundlegende Elemente des Interventionsprogramms prinzipiell anerkannt. Auf institutioneller Ebene stehen den Akteuren ganz andere Macht- und Blockademechanismen zur Verfügung. Beispielsweise kommt zu der öffentlichen Dimension auch eine nicht öffentliche Verhandlungsdimension des politischen Widerstands hinzu.

Die Wirkungen des Widerstands in Bosnien und im Kosovo sind nicht eindimensional: In Bosnien stärkt der SNSD die Autonomie der RS und hat den Einfluss der Intervenierenden begrenzt; im Kosovo erreicht VETËVENDOSJE! gerade jüngere, urbane Wähler und ist regional unabhängiger als andere Parteien. Beide fördern jedoch auch mit ihrer Rhetorik Konfrontationen mit anderen Gruppen.

Unsere Analyse zeigt, dass umfangreiche Interventionen, wie in Bosnien und im Kosovo, Widerstand erzeugen und nicht ohne diesen erwartbaren Widerstand gedacht werden können. Er ist weder zwangsläufig gewaltsam noch kann ihm stets

33 Beim SNSD als Partei in Regierungsverantwortung zeigt sich deutlich, dass die gegenüber den Intervenierenden postulierten Werte nach innen so nicht umgesetzt werden. 
nachgewiesen werden, dass er Friedensprozesse grundsätzlich ablehnt. Stattdessen nimmt Widerstand gegen die Intervention eine zentrale Rolle ein, um politische Alternativen zu formulieren. Hier wird Widerstand auch zum Ausdruck von Selbstbestimmung und Selbstverortung der Intervenierten gegenüber den unerreichbaren Intervenierenden. Widerstand ist so immer die andere Seite der Medaille und wird sich auch inhaltlich anpassen, solange die Charakteristika der Intervention unverändert bleiben. Gerade das Beispiel des Widerstands in der RS zeigt dies exemplarisch: Kaum änderten die Intervenierenden ihre Position zum Dayton-Abkommen, begannen die vormaligen Gegner des Vertrages ihn zu verteidigen. Unsere Perspektive erlaubt zudem einen neuen Blick auf die lokalen Akteure: Der Intervenierte wird politisch emanzipiert und erscheint nicht mehr als Patient oder Betreuungsfall, den die internationalen Akteure, gleich Ärzten (Free 2010; Hughes/Pupavac 2005), behandeln müssen. So kann die »Dichotomie zwischen dem handelnden intervenierenden Subjekt und dem passiven intervenierten Objekt" (Bonacker/Distler 2011: 164; Hervorh. im Orig.) weiter überwunden werden.

Hinsichtlich der Praxis von Intervention plädieren wir dafür, politischen Widerstand stärker als erwartbar und nicht per se als illegitim oder als Bedrohung zu verstehen. Wer politischen Widerstand grundsätzlich delegitimiert, anstatt die eigene Rolle für Widerstand zu reflektieren, erschwert schlussendlich Kompromisse im politischen Prozess und fördert Exklusion. Die Reflexion der Beziehung zwischen Widerstand und Intervention wäre Voraussetzung dafür, Aushandlungsprozesse direkt und explizit zum Teil des Interventionsprozesses zu machen und so auch Einfluss auf die Intensität von Widerstand ausüben zu können. Intervention ist ein politischer Prozess, der öffentliche Aushandlungsprozesse geradezu voraussetzt. 


\section{Literatur}

Albrecht, Holger 2009: Political Opposition and Authoritarian Rule in Egypt, Tübingen, in: http://nbn-resolving.de/urn:nbn:de:bsz:21-opus-42404; 17.04.2013.

Aliu, Fatmir 2012: Kosovo Police Clashes with Protesters (Balkan Insight, 22.10.2012), in: http://www.balkaninsight.com/en/article/kosovo-policeclashes-with-protesters; 05.12.2012.

Ashdown, Paddy 2002: Inaugural Speech by Paddy Ashdown, the New High Representative for Bosnia \& Herzegovina (Held at the BiH State Parliament, 27.05.2002), in: http://www.ohr.int/ohr-dept/presso/presssp/default.asp?content id $=8417 ; 06.03 .2012$.

Barnett, Michael/Zürcher, Christoph 2009: The Peacebuilder's Contract: How External Statebuilding Reinforces Weak Statehood, in: Paris, Roland/Sisk, Timothy (Hrsg.): The Dilemmas of Postwar Statebuilding: Confronting the Contradictions of Postwar Peace Operations, London, 23-52.

Bassuener, Kurt/Weber, Bodo 2010: Balkan Tango: The EU's Disjointed Policies Compound Bosnia's Paralysis, in: IP Global 2/2010, 19-25.

Bellamy, Alex J. 2004: The »Next Stage« in Peace Operations Theory?, in: International Peacekeeping 11: 1, 17-38.

Bliesemann de Guevara, Berit 2008: The State in Times of Statebuilding, in: Civil Wars 10: 4, 348-368.

Bliesemann de Guevara, Berit/Kühn, Florian P. 2010: Illusion Statebuilding. Warum der westliche Staat so schwer zu exportieren ist, Hamburg.

Bonacker, Thorsten 2010: Die Gesellschaft der Anderen. Kambodscha und die Interventionskultur der Weltgesellschaft, in: Bonacker, Thorsten/Daxner, Michael/Free, Jan H./Zürcher, Christoph (Hrsg.): Interventionskultur. Zur Soziologie von Interventionsgesellschaften, Wiesbaden, 189-218.

Bonacker, Thorsten/Distler, Werner 2011: Das Militär im Statebuilding aus interventionssoziologischer Perspektive, in: Leonhard, Nina/Werkner, Ines-Jacqueline (Hrsg.): Militärsoziologie - Eine Einführung, Wiesbaden, 158-175.

Borić, Faruk 2010: Slučaj Butmir. Bošnjačka politika između stranačkih interesa i ideologije (Puls Demokratije, 27.01.2010), in: http://arhiva.pulsdemokratije.net /index.php?id=1931\&l=bs; 27.09.2012.

Bose, Sumantra 2002: Bosnia after Dayton: Nationalist Partition and International Intervention, London.

Brand, Marcus 2003: The Development of Kosovo Institutions and the Transition of Authority from UNMIK to Local Self-Government, Genf. 
Briscoe, Ivan/Price, Megan 2011: Kosovo's New Map of Power: Governance and Crime in the Wake of Independence, in: http://www.clingendael.nl/publications /2011/20110503_cru_publication_ibriscoe.pdf; 05.03.2012.

Chandler, David 1999: Faking Democracy after Dayton, London.

Chesterman, Simon 2004: You, the People - The United Nations, Transitional Administration, and State-Building, New York, NY.

Chesterman, Simon 2007: Ownership in Theory and in Practice: Transfer of Authority in UN State-Building Operations, in: Journal of Intervention and Statebuilding 1: 1, 3-26.

Chesterman, Simon 2011: State-Building, the Social Contract, and the Death of God (Working Paper No. 11-02, Public Law and Legal Theory Research Paper Series), in: http://papers.ssrn.com/sol3/papers.cfm?abstract_id=1733905; 26.08.2013.

Daxner, Michael/Free, Jan/Bonacker, Thorsten/Zürcher, Christoph 2010: Einleitung, in: Bonacker, Thorsten/Daxner, Michael/Free, Jan H./Zürcher, Christoph (Hrsg.): Interventionskultur. Zur Soziologie von Interventionsgesellschaften, Wiesbaden, 7-18.

Dean, Robert 2007: Final Report of the Special Prosecutor to the SRSG, in: http:// www.cpt.coe.int/documents/srb/2009-03-inf-eng.htm; 02.07.2007.

Distler, Werner 2010: Die Bedingungen der Intervention. Interaktion in einer Ausnahmesituation, in: Bonacker, Thorsten/Daxner, Michael/Free, Jan H. Free/Zürcher, Christoph (Hrsg.): Interventionskultur. Zur Soziologie von Interventionsgesellschaften, Wiesbaden, 119-140.

Donais, Timothy 2006: The Limits of Post-conflict Police Reform, in: Innes, Michael A. (Hrsg.): Bosnian Security After Dayton. New Perspectives, New York, NY, 173-190.

Džihić, Vedran 2007: Dilemmata im Prozess der »Europäisierung« Bosniens (Working Paper Series, Paper No. 1, Center for European Integration Strategies), in: http://www.ceis-eu.org/publications/working_papers/2007/ceis_wps_no1. pdf; 21.10.2009.

Džihić, Vedran 2009: Multiple Faces of the Bosnian »Crisis Circle«: Ethnonationalism and Ethnopolitics in Post-Dayton-Bosnia and their Effects on Democratization, in: Felberbauer, Ernst/Jureković, Predrag/Labarre, Frédéric (Hrsg.): Workshop of the Study Group, PfP Consortium, Wien, 31-55.

ESI - European Stability Initiative 1999: Reshaping International Priorities in Bosnia and Herzegovina. Part One: Bosnian Power Structures, Berlin.

Esser, Hartmut 1999: Soziologie. Spezielle Grundlagen. Band 5: Institutionen, Frankfurt a. M. 
Falconer-Stout, Zachariah James 2009: The Political Economy of Sub-State Development in Post-Conflict Bosnia and Herzegovina (MA Thesis at the Department of International Relations and European Studies, Central European University), in: www.etd.ceu.hu/2009/falconer-stout_zachariah.pdf; 10.04.2012.

Foucault, Michel 1977: Der Wille zum Wissen. Sexualität und Wahrheit I, Frankfurt a. M.

Free, Jan H. 2010: Wege zu einer Soziologie moderner Friedenseinsätze, in: Bonacker, Thorsten/Daxner, Michael/Free, Jan H./Zürcher, Christoph (Hrsg.): Interventionskultur. Zur Soziologie von Interventionsgesellschaften, Wiesbaden, 49-74.

Gavrić, Saša/Banović, Damir 2007: Verantwortung übernehmen? - Wahlen und Regierungsbildung in Bosnien und Herzegowina, in: Südosteuropa Mitteilungen 47: 2, 52-64.

Gromes, Thorsten 2007: Demokratisierung nach Bürgerkriegen. Das Beispiel Bosnien und Herzegowina, Frankfurt a. M.

Guilhot, Nicolas 2005: The Democracy Makers: Human Rights and International Order, New York, NY.

Hays, Don/Crosby, Jason 2006: From Dayton to Brussels: Constitutional Preparations for Bosnia's EU Accession, Washington, D.C.

Hughes, Caroline/Pupavac, Vanessa 2005: Framing Post-Conflict Societies: International Pathologisation of Cambodia and the Post-Yugoslav States, in: Third World Quarterly 26: 6, 873-889.

Human Rights Watch 2007: Better Later than Never: Enhancing the Accountability of International Institutions in Kosovo, in: http://www.hrw.org/sites/default/file s/reports/kosovo0607web.pdf; 05.03.2012.

ICG - International Crisis Group 2003: Bosnia's Nationalist Governments: Paddy Ashdown and the Paradoxes of State Building (Balkans Report No. 146), Sarajevo.

ICG - International Crisis Group 2005: Bosnia's Stalled Police Reform: No Progress, No EU (Europe Report No. 164), Sarajevo.

ICG - International Crisis Group 2007: Ensuring Bosnia's Future: A New International Engagement Strategy (Europe Report No. 180), Sarajevo.

ICG - International Crisis Group 2011 a: Bosnia: Europe's Time to Act (Europe Briefing No. 59), Sarajevo.

ICG - International Crisis Group 2011 b: Bosnia: What Does Republika Srpska

Want? (Europe Report No. 214), Sarajevo. 
International Civilian Office 2012: News Release, 12.01.2012, Pristina.

Izborna Komisija Bosna i Hercegovina 2002 a: Opći izbori 2002 - konaćni rezultati.

Izborna utrka: 501. Zastupnički/Predstavnički Dom Parlamentarne Skupštine Bosne i Hercegovine, in: http://www.izbori.ba/Documents/Documents/Rezultat i\%20izbora\%2096-2002/Rezultati2002/Puni/PartijskiGlasoviEntitet-501.pdf; 28.03.2013.

Izborna Komisija Bosna i Hercegovina 2002 b: Opći izbori 2002 - konaćni rezultati. Izborna utrka: 502. Zastupnički/Predstavnički Dom Parlamentarne Skupštine Bosne i Hercegovine, in: http://www.izbori.ba/Documents/Documents/Rezultat i\%20izbora\%2096-2002/Rezultati2002/Puni/PartijskiGlasoviEntitet-502.pdf; 28.03.2013.

Izborna Komisija Bosna i Hercegovina 2006: Zastupnički/Predstavnički Dom Parlamentarne Skupštine Bosne i Hercegovine, in: http://www.izbori.ba/rezultati/ konacni/parlament_bih/index.htm\#; 28.03.2013.

Kallaba, Pëllumb/Ferati, Violeta 2012: Mapping the UNSCR 1244 Legacy in PostIndependence Kosovo: Between Contestation and Recognition, Pristina.

KIPRED - Kosovar Institute for Policy Research and Development 2011: Kosovo

National Elections 2010: Overview and Trends, in: http://www.fes-prishtina.org /wb/media/Publications/2011/KIPRED\%20-\%20Parliamentary\%20Elections \%202010\%20\%28English\%29.pdf; 06.03.2012.

Knaus, Gerald/Martin, Felix 2003: Travails of the European Raj, in: Journal of Democracy 14: 3, 60-74.

Knoll, Bernhard 2007: Legitimacy and UN Administration of Territory, in: Journal of International Law and Policy 4: 1, 1-15.

Kovačević, Ljiljana 2011:RS'sDodikStickstoReferendumPlanDespiteInternational Condemnation (Southeast European Times, 05.05.2011), in: http://www.setimes .com/cocoon/setimes/xhtml/en_GB/features/setimes/features/2011/05/05/featu re-02; 08.05.2011.

Krasner, Stephen D. 2007: Alternativen zur Souveränität. Neue Institutionen für kollabierte und scheiternde Staaten, in: Beisheim, Marianne/Folke Schuppert, Gunnar (Hrsg.): Staatszerfall und Governance, Baden-Baden, 163-173.

Kurti, Albin 2010: Objections Against the Panel, Vetëvendosje!, 14.06.2010, in: http://www.vetevendosje.org/?cid=2,65,4001; 06.03.2012.

Kurti, Albin 2011: JISB Interview: Kosova in Dependence: From Stability of Crisis to the Crisis of Stability, in: Journal of Intervention and Statebuilding 5: 1, 89-97. Lindvall, Daniel 2009: The Limits of the European Vision in Bosnia and Herzegovina: An Analysis of the Police Reform Negotiations, Stockholm. 
Loza, Tihomir 2007: Watch Out, Milorad (Transitions Online, 01.09.2007), in: http:// www.ceeol.com/aspx/issuedetails.aspx?issueid $=49 \mathrm{e} 3 \mathrm{a} 2 \mathrm{~d} 7-1 \mathrm{~b} 6 \mathrm{a}-48 \mathrm{e} 7-9 \mathrm{~d} 98-90$ 6e8292e29e\&articleId=7eea77e7-4ec7-449f-ae78-63be03d94bb9;26.08.2013.

Luhmann, Niklas 1989: Theorie der politischen Opposition, in: Zeitschrift für Politik 36: 1, 13-26.

Mac Ginty, Roger 2011: International Peacebuilding and Local Resistance: Hybrid Forms of Peace, New York, NY.

Narten, Jens 2007: Dilemmas of Promoting Local Ownership: Statebuilding in Postwar Kosovo, in: Paris, Roland/Sisk, Timothy (Hrsg.): The Dilemmas of Statebuilding. Confronting the Contradictions of Postwar Peace Operations, 252-284.

Newman, Edward/Richmond, Oliver 2006: The Impact of Spoilers on Peace Processes and Peacebuilding (Policy Briefing No. 2/2006), in: http://reliefweb.int/ sites/reliefweb.int/files/reliefweb_pdf/node-23321.pdf; 06.03.2012.

Nilsson, Desirée/Söderberg Kovacs, Mimmi 2011: Revisiting an Elusive Concept:

A Review of the Debate on Spoilers in Peace Processes, in: International Studies Review 13: 4, 606-626.

Office of the Prime Minister, Republic of Srpska Government 2011: The 5th Report of Republika Srpska to the United Nations Security Council: Reply to High Representative's Letter and Special Report of 4 May 2011 to the UNSC, Banja Luka.

Office of the Prime Minister 2012 a: Press Release, 14.01.2012, in: http://www.kry eministri-ks.net/index.php?page=2,9,2598; 06.03.2012.

Office of the Prime Minister 2012 b: Press Release, 16.01.2012, in: http://www.kry eministri-ks.net/index.php?page=2,9,2599; 06.03.2012.

Office of the Prime Minister 2012 c: Press Release, 27.02.2012, in: http://www.kry eministri-ks.net/index.php?page=2,9,2691; 06.03.2012.

Paris, Roland 2004: At War's End: Building Peace after Civil Conflict, Cambridge, MA.

Paris, Roland 2010: Saving Liberal Peacebuilding, in: Review of International Studies 36: 2, 337-365.

Parish, Matthew 2007: The Demise of the Dayton Protectorate, in: Journal of Intervention and Statebuilding 1: Special Supplement, 11-23.

PIC - Peace Implementation Council 2012: The Peace Implementation Council and its Steering Board, 16.02.2012, in: http://www.ohr.int/pic/default.asp?content_ $\mathrm{id}=38563 ; 17.04 .2013$. 
Pilsel, Drago 2009: The Role of Media in the Process of Peace-Building, in: Felberbauer, Ernst/Jureković, Predrag/Labarre, Frédéric (Hrsg.): Workshop of the Study Group, PfP Consortium, Wien, 177-182.

Pouligny, Béatrice 2006: Peacekeeping Seen from Below: UN Missions and Local People, London.

Pugh, Michael 2000: Introduction: The Ownership of Regeneration and Peacebuilding, in: Pugh, Michael (Hrsg.): Regeneration of War-Torn Societies, London, 1-14.

Rawski, Frederick 2002: To Waive or Not to Waive: Immunity and Accountability in U.N. Peacekeeping Operations, in: Connecticut Journal of International Law 18: 1, 103-132.

Richmond, Oliver P. 2009: A Post-Liberal Peace: Eirenism and the Everyday, in: Review of International Studies 35: 3, 557-580.

Richmond, Oliver P./Franks, Jason 2009: Liberal Peace Transitions, Edinburgh.

Richmond, Oliver/Mitchell, Audra 2011: Peacebuilding and Critical Forms of Agency: From Resistance to Subsistence, in: Alternatives: Global, Local, Political 36: 4, 326-344.

Roberts, David 2011: Liberal Peacebuilding and Global Governance: Beyond the Metropolis, London.

Sebastian, Sofia 2007: Leaving Dayton Behind: Constitutional Reform in Bosnia and Herzegovina, in: http://www.ustavnareforma.ba/en/amendment_proposals/ academic_community/sofia_sebastian_leaving_dayton_behind_constitutional_ reform in bosnia and herzegovina fride working paper 46.html; 10.12.2012.

SNSD o.J.: Program Saveza Nezavisnih Socijaldemokrata (Programm der Unabhängigen Sozialdemokraten), in: http://www.snsd-bileca.org/doc/snsd_program za_web.pdf; 16.09.2013.

Stedman, Stephen J. 1997: Spoiler Problems in Peace Processes, in: International Security 22: 2, 5-53.

Steffek, Jens 2003: The Legitimation of International Governance: A Discourse Approach, in: European Journal of International Relations 9: 2, 249-275.

Stewart, Rory/Knaus, Gerald 2011: Can Intervention Work?, New York, NY.

Talentino, Andrea K. 2007: Perceptions of Peacebuilding: The Dynamic of Imposer and Imposed Upon, in: International Studies Perspectives 8: 2, 152-171.

UNDP - United Nations Development Programme BiH 2006: Early Warning System Research 2000-2006, Sarajevo.

UNDP - United Nations Development Programme BiH 2007: Early Warning System Annual Report 2007, Sarajevo. 
UNDP - United Nations Development Programme BiH 2008: Early Warning System. Fourth Quarterly Report - December 2008, Sarajevo.

UNMIK Press Briefing 2007: Transcript of Press Briefing by Special Prosecutor Robert Dean on his Interim Report to the SRSG Regarding the Deaths and Serious Wounding of Protestors during the 10 February 2007 Demonstration in Pristina, in: http://pfpk.wikispaces.com/file/view/Press+Release+Regarding+ Romanian+FPU.pdf; 06.03.2012.

United Nations Security Council 2007: Report of the Secretary-General on the United Nations Interim Administration Mission in Kosovo (S/2007/134), in: http://www.unmikonline.org/SGReports/S-2007-134.pdf; 29.01.2012.

United Nations Security Council 2012: Report of the Secretary-General on the United Nations Interim Administration Mission in Kosovo (S/2012/72), in: http://www.unmikonline.org/SGReports/S-2012-72.pdf; 06.03.2012.

Venice Commission 2005: Opinion on the Constitutional Situation in Bosnia and Herzegovina and the Powers of the High Representative, Venedig.

Vetëvendosje! 2006: Newsletter from the Movement for SELF-DETERMINATION! (No. 1), in: http://www.vetevendosje.org/repository/docs/newsletter_1. pdf; 05.03.2012.

Vetëvendosje! 2010a: The Manifesto Principles and Priorities of Lëvizja VETËVENDOSJE!: 100 Points Joined Together by the Changes Necessary for Kosova, in: http://www.vetevendosje.org/repository/docs/Programi_i_shkurte_ anglisht.pdf; 05.03.2012.

Vetëvendosje! 2010 b: Movement's Manifesto, in: http://www.vetevendosje.org/rep ository/docs/Movement\%27s_Manifesto.pdf; 05.03.2012.

Vetëvendosje! 2010 c: Why No Negotiations?, in: http://www.vetevendosje.org/rep ository/docs/Why_no_negotiations.pdf; 05.03.2012.

Vetëvendosje! $2010 \mathrm{~d}$ : History of Lëvizja VETËVENDOSJE!, in: http://www.vetev endosje.org/repository/docs/index3.pdf; 05.03.2012.

Vetëvendosje! 2010 e: Deconstruction of the Declaration of Independence, in: http: //www.vetevendosje.org/repository/docs/Deconstruction_of_the_Declaration of_Independence.pdf; 05.03.2012.

Vetëvendosje! 2012: Newsletter from the Movement for SELF-DETERMINATION!, in: http://www.vetevendosje.org/repository/docs/Newsletter_Nr. 292.pdf; 06.03.2012.

Visoka, Gezim 2011: International Governance and Local Resistance in Kosovo:

The Thin Line between Ethical, Emancipatory and Exclusionary Politics, in: Irish Studies in International Affairs 22: 1, 99-125. 
Visoka, Gezim 2012: The »Kafkaesque Accountability« of International Governance in Kosovo, in: Journal of Intervention and Statebuilding 6: 2, 189-212.

Wesley, Michael 2008: The State of the Art on the Art of State Building, in: Global Governance 14: 3, 369-385.

Zaum, Dominik 2007: The Sovereignty Paradox: The Norms and Politics of International Statebuilding, Oxford.

Zürcher, Christoph/Koehler, Jan 2003: Introduction: Potentials of Disorder in the Caucasus and Yugoslavia, in: Zürcher, Christoph/Koehler, Jan (Hrsg.): Potentials of Disorder, Manchester, 1-22.

Zürcher, Christoph/Manning, Carrie/Evenson, Kristie/Hayman, Rachel/Riese, Sarah/Roehner, Nora 2013: Costly Democracy: Peacebuilding and Democratization after War, Stanford, CT, i. E.

\section{Die AutorInnen}

Werner Distler, M. A., ist wissenschaftlicher Mitarbeiter am Zentrum für Konfliktforschung (ZfK), Philipps-Universität Marburg.

E-Mail: werner.distler@staff.uni-marburg.de

Sarah Riese hat am Fachbereich Politik- und Sozialwissenschaften der Freien Universität (FU) in Berlin promoviert und arbeitet im Jugendbildungsbereich in Südosteuropa.

E-Mail: sriese@zedat.fu-berlin.de 